\title{
Accurate Thermodynamics for Short-Ranged Truncations of Coulomb Interactions in Site-Site Molecular Models
}

\author{
Jocelyn M. Rodger:* \\ Institute for Physical Science and Technology, University of Maryland, College Park, Maryland 20742 and \\ Chemical Physics Program, University of Maryland, College Park, Maryland 20742 \\ John D. Week $₫$ \\ Institute for Physical Science and Technology, University of Maryland, College Park, Maryland 20742 and \\ Department of Chemistry and Biochemistry, University of Maryland, College Park, Maryland 20742
}

(Dated: July 2, 2018)

\begin{abstract}
Coulomb interactions are present in a wide variety of all-atom force fields. Spherical truncations of these interactions permit fast simulations but are problematic due to their incorrect thermodynamics. Herein we demonstrate that simple analytical corrections for the thermodynamics of uniform truncated systems are possible. In particular results for the SPC/E water model treated with spherically-truncated Coulomb interactions suggested by local molecular field theory [Proc. Nat. Acad. Sci. USA 105, 19136 (2008)] are presented. We extend results developed by Chandler [J. Chem. Phys. 65, 2925 (1976)] so that we may treat the thermodynamics of mixtures of flexible charged and uncharged molecules simulated with spherical truncations. We show that the energy and pressure of spherically-truncated bulk SPC/E water are easily corrected using exact second-moment-like conditions on long-ranged structure. Furthermore, applying the pressure correction as an external pressure removes the density errors observed by other research groups in NPT simulations of spherically-truncated bulk species.
\end{abstract}

\section{INTRODUCTION}

Most classical intermolecular potential models assign effective point charges to intramolecular interaction sites to describe charge separation in polar molecules and the ability to form hydrogen bonds 1.2 . Thus, even in purely neutral systems, charge-charge interactions remain important and expensive components of molecular simulations, usually dealt with via Ewald summations or some other lattice-sum-like technique ${ }^{3}$.

Recently there has been renewed interest in spherically truncating the $1 / r$ interaction and neglecting the longranged components beyond a specified cutoff radius $4,5,6,7$. This permits fast and efficient simulations that scale linearly with system size. However, spherical truncation are problematic to implement for Coulomb interactions. While many groups have found that accurate local pair correlation functions in uniform systems may be obtained by a variety of spherical truncations of $1 / r^{4}, 5,6,7,8$, two common and valid objections to spherical truncations remain:

1. they fail for structural and electrostatic properties in nonuniform systems, e.g., systems with point charges confined between walls $\frac{9,10}{}$, and

2. in uniform systems, the thermodynamics predicted by such truncations ${ }^{3}$ and even the bulk densities in NPT simulations ${ }^{7,11}$ are known to be inaccurate.

Recently we overcame the first objection, showing that local molecular field (LMF) theory ${ }^{12,13}$ provides an accurate path to structural properties in both ionic and aqueous nonuniform systems using a spherical truncation of $1 / r$ along with a restructured external potential $\mathcal{V}_{\mathrm{R}}$ to

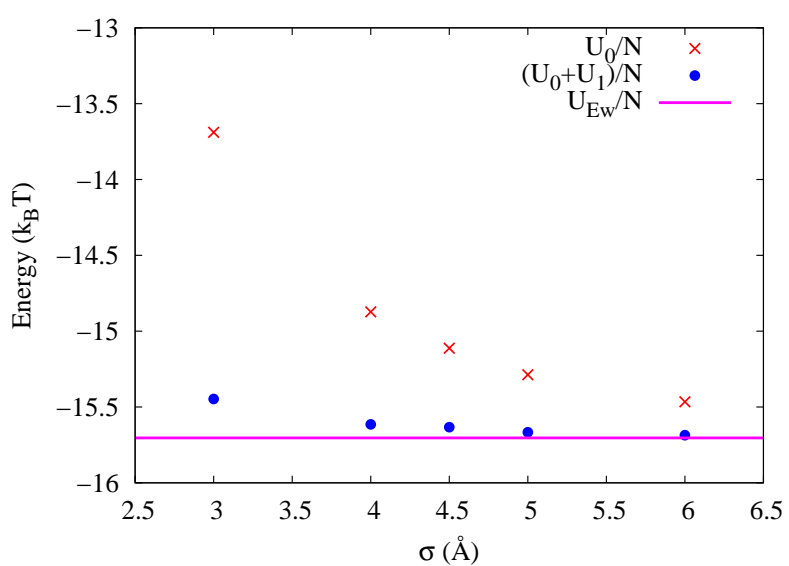

FIG. 1: Plot of total potential energy without $\left(U_{0} / N\right.$ in red crosses) and with the long-range correction $\left(U_{0} / N+U_{1} / N\right.$ in blue circles) for the full range of $\sigma$ studied, representing greater inclusion of nearby core interactions. The length $\sigma$ sets the scale for the smooth truncation of the Coulomb interactions as is explained further in Section [II Error bars are smaller than the data points. The Ewald determined energy is indicated by a horizontal line.

account for the net averaged effects of the long-ranged forces neglected in the spherical truncation ${ }^{14,15,16}$. Electrostatic properties are then also very accurately described 16,17 .

Generalizing previous work for purely ionic systems ${ }^{18,19}$, we show here that the LMF framework also guides us to simple analytic corrections for the energy and pressure of a general uniform mixture of both polar and charged site-site molecules. As in thermodynamic 
perturbation theory $\stackrel{20}{ }$, we can view a short-ranged truncation of $1 / r$ as the reference system for the fully interacting system. Our corrections are appropriate and accurate only for certain special well-chosen reference systems as discussed below, which we refer to as "mimic systems". The total energy and pressure of the full system is then given by the sums

$$
\begin{aligned}
& U_{\text {tot }}=U_{0}+U_{1} \\
& P_{\text {tot }}=P_{0}+P_{1} .
\end{aligned}
$$

Typical simulations using such a spherical truncation of the $1 / r$ interaction yield only $U_{0}$ and $P_{0}$, and our task is to determine the corrections $U_{1}$ and $P_{1}$ that would arise from an accurate treatment of the long-ranged interactions.

In particular, as shown in Fig. 1 for bulk SPC/E water $21, U_{0}$ alone over a wide range of truncation distances parametrized by the length $\sigma$ does not agree with the energy as calculated using three-dimensional Ewald sums. In contrast, any $\sigma$ of $3.0 \AA$ or greater reproduces the short-ranged pair correlations predicted by Ewald sums quite well 16,22 . As $\sigma$ increases, the energies are in better agreement with the Ewald calculated values, but noticeable differences remain even for large $\sigma$.

Related problems arose in recent NPT ensemble simulations of water ${ }^{7,11}$. Using Wolf sums ${ }^{5}$, researchers simulated systems quite similar to the truncated system dictated by LMF theory for $\sigma \approx 5.0 \AA$ (labeled " $\mathrm{DFS}_{2}$ " in Ref. 7) and for a range of $\sigma$ in Ref. 11. Each used the NPT ensemble with a pressure of $1 \mathrm{~atm}$. They found generally good structural agreement, but noted thermodynamic discrepancies, like an elevated energy and a depressed density as compared to Ewald simulations.

Here we use LMF theory along with long-wavelength constraints on the behavior of charge correlation functions implied by exact expressions for the dielectric constant in neutral systems ${ }^{23}$ and the related StillingerLovett moment conditions $\underline{24}$ for ionic systems to derive analytic expressions for $U_{1}$ and $P_{1}$. Results incorporating this correction for the energy of SPC/E water are also given in Fig. 1 and their high accuracy is evident.

\section{LOCAL MOLECULAR FIELD THEORY FOR SITE-SITE MOLECULAR SIMULATIONS}

Local molecular field theory provide a general theoretical framework for assessing and correcting spherical truncations of Coulomb interactions. The derivation of LMF theory for systems with Coulomb interactions has been recently reviewed elsewhere ${ }^{13}$, and we will be brief in our discussion here.

LMF theory divides the $1 / r$ potential into short- and long-ranged parts characterized by the length $\sigma$ as

$$
\frac{1}{r}=v_{0}(r)+v_{1}(r)=\frac{\operatorname{erfc}(r / \sigma)}{r}+\frac{\operatorname{erf}(r / \sigma)}{r} .
$$

This potential separation isolates strong short-ranged and rapidly-varying Coulomb interactions in $v_{0}(r)$, while the remaining slowly-varying long-ranged forces are contained in $v_{1}(r) . \quad v_{1}(r)$ is proportional to the electrostatic potential arising from a smooth normalized Gaussian charge distribution with width $\sigma$, and is defined by the convolution

$$
v_{1}(r) \equiv \frac{1}{\pi^{3 / 2} \sigma^{3}} \int e^{-r^{\prime 2} / \sigma^{2}} \frac{1}{\left|\mathbf{r}-\mathbf{r}^{\prime}\right|} d \mathbf{r}^{\prime} .
$$

By construction, $v_{1}(r)$ is slowly-varying in $r$-space over the smoothing length $\sigma$ (see, e.g., Fig. 1 in Ref. 13), and contains only small wave vectors in reciprocal space, as can be seen from its Fourier transform

$$
\hat{v}_{1}(k)=\frac{4 \pi}{k^{2}} \exp \left[-(k \sigma)^{2} / 4\right] .
$$

The short-ranged $v_{0}(r) \equiv 1 / r-v_{1}(r)$ is then the screened potential resulting from a point charge surrounded by a neutralizing Gaussian charge distribution whose width $\sigma$ also sets the scale for the smooth truncation of $v_{0}$. At distances much less than $\sigma$ the force from $v_{0}(r)$ approaches that from the full $1 / r$ potential.

Starting from the exact Yvon-Born-Green hierarchy 20 and exploiting the slowly-varying nature of $v_{1}(r)$, LMF theory accounts for the averaged effects of the longranged component $v_{1}(r)$ in a mean-field sense by a rescaled, self-consistent, mean electrostatic potential $\mathcal{V}_{\mathrm{R}}(\mathbf{r})$. The short-ranged $v_{0}(r)$, with $\sigma$ chosen large enough to capture relevant nearest-neighbor interactions like core repulsions and hydrogen bonding, is the spherical truncation used in LMF theory. A system in the presence of $\mathcal{V}_{\mathrm{R}}(\mathbf{r})$ with $1 / r$ replaced by the short-ranged $v_{0}(r)$ is referred to as a mimic system, and densities associated with such a system are indicated by $\rho_{R}(\mathbf{r})$.

Often for uniform systems, $\mathcal{V}_{\mathrm{R}}(\mathbf{r})$ has negligible effect on short-ranged pair correlations ${ }^{18,22}$. For such uniform systems, we may simulate simply using $v_{0}(r)$ with $\mathcal{V}_{\mathrm{R}}(\mathbf{r})=0$ and thus generate densities $\rho_{0}(\mathbf{r})$. We call this approximation to the full LMF theory the strong-coupling approximation and refer to the resulting truncated water model as Gaussian-truncated water. While not generally true, LMF theory in the strong-coupling approximation is related to other spherical truncations such as site-site reaction field ${ }^{8}$ and Wolf truncations ${ }^{5}$.

LMF theory seeks to obtain the properties of the full, uniform system from the simulation of the shortranged system, whose total energy $U_{0}$ for Gaussiantruncated SPC/E water includes all contributions from the Lennard-Jones interactions as well as the shortranged components of the Coulomb interactions due to $v_{0}(r)$. Other authors have used the $v_{0}(r)$ truncation and proposed numerical corrections to the energy and pressure of ionic systems based on integral equation meth$\mathrm{ods}^{25}$, but the simple and accurate analytical corrections possible using moment conditions and our choice of $v_{0}(r)$ and $v_{1}(r)$ as described below in Sections [IV and $\mathrm{V}$ have not been previously derived. 


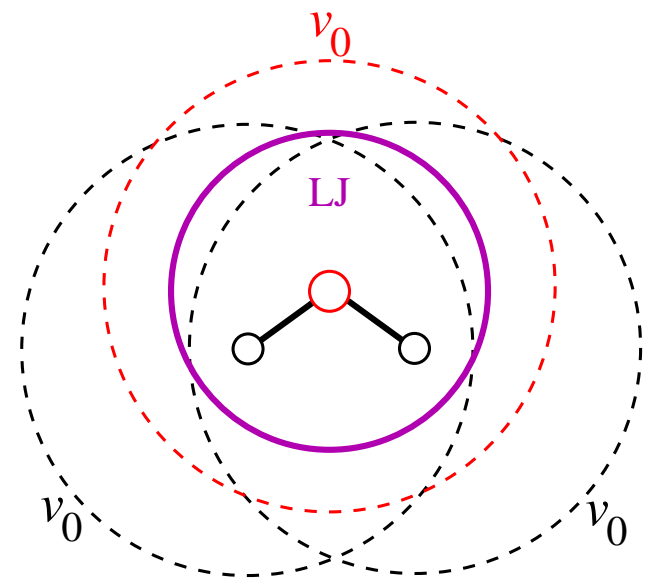

FIG. 2: Diagram of Gaussian-truncated SPC/E water. The traditional SPC/E model of water is shown in the center with three point charges and a Lennard-Jones core to represent the excluded volume. Gaussian-truncated water is constructed by replacing the three point charges with the corresponding short-ranged $v_{0}(r)$.

\section{SIMULATION DETAILS}

The main result of this paper is a general derivation of simple analytical corrections for the spherical truncation of Coulomb interactions in simple charged and uncharged site-site molecular models. In order to demonstrate the accuracy of these corrections, we also have carried out a series of simulations of a molecular water model at ambient conditions.

The water model we choose is $\mathrm{SPC} / \mathrm{E}$ water ${ }^{21}$, shown in the center of Fig. 2. A Lennard-Jones core, depicted by the solid circle with diameter $\sigma_{L J}=3.161 \AA$ accounts for the excluded volume of the molecules, and point charges are present at each of the atomic sites in order to represent the charge separation along the $\mathrm{OH}$ bonds and to allow for hydrogen bonding between molecules. In order to simulate Gaussian-truncated water, we replace the $1 / r$ interaction from each of these point charges by the shortranged $v_{0}(r)$ as represented by the dashed circles drawn here to scale with diameter $\sigma=4.5 \AA$.

We carried out a molecular dynamics simulation of a uniform system of $1728 \mathrm{SPC} / \mathrm{E}$ water molecules using a modified version of DLPOLY2.16 26 . The Berendsen thermostat ${ }^{27}$ with a relaxation time constant of $0.5 \mathrm{ps}$ is used to maintain the temperature at $300 \mathrm{~K}$, and, for the final set of data presented, a Berendsen barostat maintains the pressure. All simulations use a timestep of $1 \mathrm{fs}$. For the spherical truncations, $\sigma$ ranges from $3.0 \AA$ to $6.0 \AA$, with the cutoff radius ranging from $9.5 \AA$ (the cutoff radius for the Lennard-Jones core) to $13.5 \AA$. As explored in Refs. 16 and 22 , any $\sigma$ of $3.0 \AA$ or greater reproduces the short-ranged pair correlations predicted by Ewald sums quite well. For our benchmark, we compare to simulations using three-dimensional Ewald sums with $\alpha=0.30 \AA^{-1}$ and $k_{\max }=10$. The systems were each equilibrated for a total of 500 ps, with $1.5 \mathrm{~ns}$ of data collection; error bars were based on 100 ps blocks of data.

\section{ANALYTICAL ENERGY CORRECTION VIA MOMENT CONDITIONS}

We assume here that charged interactions arise only between charges on different molecules, as is the case for typical molecular liquid models like SPC/E water. These ideas can be extended to larger molecular species with intramolecular charge-charge interactions between furtherneighbor sites, as briefly discussed in Appendix A

The total Coulomb energy $U^{q}$ for the full system can then be exactly expressed in terms of a two-point intermolecular charge-density function $\rho^{q q}$ (with units charge $^{2} /$ volume $^{2}$ ) as

$$
\begin{aligned}
U^{q} & =\frac{1}{2} \int d \mathbf{r} \int d \mathbf{r}^{\prime} \frac{\rho^{q q}\left(\mathbf{r}, \mathbf{r}^{\prime}\right)}{\left|\mathbf{r}-\mathbf{r}^{\prime}\right|} \\
& =\frac{V}{2} \int d \mathbf{r} \rho^{q q}(r) v_{0}(r)+\frac{V}{2} \int d \mathbf{r} \rho^{q q}(r) v_{1}(r)
\end{aligned}
$$

where we have used the uniformity of the fluid and Eq. (2) in the second equality. The composite function $\rho^{q q}(r)$ is a charge-weighted linear combination of all intermolecular, two-point site-site distribution functions ${ }^{23,28}$, and a detailed expression is given in Appendix A.

As in Ref. 19, we argue that the first term on the right in Eq. (5) can be accurately approximated by $U_{0}^{q}$, the energy obtained directly from the Gaussian-truncated water simulation using $v_{0}(r)$ alone, because at short distances where $v_{0}(r)$ is non-negligible, $\rho_{0}^{q q}(r)$ closely resembles the exact $\rho^{q q}(r)$. Thus we have

$$
\frac{U^{q}}{V} \approx \frac{1}{2} \int d \mathbf{r} \rho_{0}^{q q}(r) v_{0}(r)+\frac{1}{2} \int d \mathbf{r} \rho^{q q}(r) v_{1}(r) .
$$

However, as noted in Ref. 18, a similar approximation for the second term will fail because $v_{1}(r)$ mainly contains small-wavevector components, exactly the range of $k$-components where $\rho_{0}^{q q}(r)$ will not accurately represent the $\rho^{q q}(r)$ of the full system. In fact, this integral will diverge if constraints due to neutrality in ionic systems are not obeyed. Similar considerations are true for the mixed molecular systems considered here.

Thus, we again follow the more fruitful path of writing the second term in $k$-space and approximating the long wavelength behavior of the charge energy function based on exact relations. For a uniform system, we may use Parseval's relation and Eq. (4) to reexpress Eq. (6) exactly as

$$
\frac{U^{q}}{V} \approx U_{0}^{q}+\frac{1}{2} \frac{1}{(2 \pi)^{3}} \int d \mathbf{k} \frac{4 \pi}{k^{2}} \hat{\rho}^{q q}(k) e^{-k^{2} \sigma^{2} / 4} .
$$

This choice in Eq. (4) of $v_{1}(r)$ in LMF theory allows us to make a highly useful approximation that highlights its advantages over other possible potential separations. The Gaussian from $\hat{v}_{1}(k)$ damps out the large 
$k$-contributions to the second term, $U_{1}^{q}$. Thus for sufficiently large $\sigma$ we can simply represent $\hat{\rho}^{q q}(k)$ by its two smallest moments in $k$-space,

$$
\hat{\rho}^{q q}(k) \approx \hat{\rho}^{(0) q q}+\hat{\rho}^{(2) q q} k^{2}+\mathcal{O}\left(k^{4}\right) .
$$

We show in Appendix A that $\hat{\rho}^{q q}(k)$ is very simply related to the basic charge-charge linear response function $\hat{\chi}^{q q}(k)$ that appears in Chandler's formula 23 for the dielectric constant of a neutral molecular mixture. We have generalized the derivation to include both neutral and charged molecular species in Appendix $\mathrm{A}$ and demonstrate the simpler expansion of $\hat{\chi}^{q q}(k)$ in Appendix B. This expression is a consequence of Stillinger-Lovett-like sum rules ${ }^{20,29}$ arising from the assumption that the potential induced by a test charge $Q$ in a uniform molecular fluid approaches $4 \pi Q / \epsilon k^{2}$ at small $k$ to linear order in $Q$. This allows us to relate the moments of $\hat{\rho}^{q q}$ to the dielectric constant $\epsilon$ and other molecular properties. A system with mobile ions exhibits complete screening with $\epsilon=\infty$.

Here we simply state the final expansion of the twopoint charge-density function up to second order in $k$, as derived in Appendix A. We find for a general mixture of charged $(\mathrm{C})$ and neutral $(\mathrm{N})$ polarizable site-site molecules without intramolecular charge-charge interactions,

$$
\begin{aligned}
\hat{\rho}^{q q}(k)= & -\sum_{C} \rho_{C} q_{C}^{2}+k^{2} \frac{\mathrm{k}_{\mathrm{B}} \mathrm{T}}{4 \pi} \frac{\epsilon-1}{\epsilon} \\
& -k^{2} \sum_{N} \rho_{N}\left\{\frac{1}{3} \mu_{N}^{2}+\mathrm{k}_{\mathrm{B}} \mathrm{T} \alpha_{N}\right\} \\
& +\frac{1}{6} k^{2} \sum_{C} \rho_{C} \sum_{\alpha, \gamma} q_{\alpha C} q_{\gamma C}\left\langle l_{\alpha \gamma C}^{2}\right\rangle+\mathcal{O}\left(k^{4}\right) .
\end{aligned}
$$

Here, $\rho_{C}$ and $\rho_{N}$ are charged and neutral species bulk densities, $\mu_{N}$ indicates the dipole moment of a neutral molecule, and $\alpha_{N}$ is the molecular polarizability. The final term sums over the the average of the square of given bond lengths $l_{\alpha \gamma C}$ in a charged molecule. For larger CHARMM- or AMBER-like molecular models, a generalization of this approach leading to related moment-like conditions is possible.

Using this small-moment expansion in Eq. (7) and noting that the integrals of Gaussians involved can be analytically evaluated, we find

$$
\begin{aligned}
\frac{U_{1}^{q}}{V} \approx-\frac{1}{\sigma \sqrt{\pi}} & \sum_{C} \rho_{C} q_{C}^{2}+\frac{2}{\sigma^{3} \sqrt{\pi}} \frac{\mathrm{k}_{\mathrm{B}} \mathrm{T}}{4 \pi} \frac{\epsilon-1}{\epsilon} \\
& -\frac{2}{\sigma^{3} \sqrt{\pi}} \sum_{N} \rho_{N}\left\{\frac{1}{3} \mu_{N}^{2}+\mathrm{k}_{\mathrm{B}} \mathrm{T} \alpha_{N}\right\} \\
& +\frac{1}{3 \sigma^{3} \sqrt{\pi}} \sum_{C} \rho_{C} \sum_{\alpha, \gamma} q_{\alpha C} q_{\gamma C}\left\langle l_{\alpha \gamma C}^{2}\right\rangle .
\end{aligned}
$$

In particular, for bulk SPC/E water, which is neutral and nonpolarizable, we have

$$
\frac{U_{1}^{q}}{N} \approx \frac{2}{\sigma^{3} \sqrt{\pi}}\left(\frac{\mathrm{k}_{\mathrm{B}} \mathrm{T}}{4 \pi \rho_{w}} \frac{\epsilon_{w}-1}{\epsilon_{w}}-\frac{\mu_{w}^{2}}{3}\right)
$$

where $\rho_{w}$ is the bulk density, $\epsilon_{w}$ is the dielectric constant, and $\mu_{w}$ is the dipole moment of SPC/E water. In contrast to the expression developed for ionic solutions ${ }^{18.19}$, the energy correction now incorporates a significant, nontrivial contribution from the dipole moment.

In fact $U_{1}^{q} / N$ is negative and may be bounded from above as $U_{1}^{q} / N \leq-118.3 \frac{\mathrm{kJ}}{\mathrm{mol}} \cdot \AA^{3} / \sigma^{3}$ for $T=300 \mathrm{~K}$, by assuming $\epsilon \rightarrow \infty$ and using $\mu_{w}$ determined from the rigid geometry of the SPC/E water molecule. In obtaining this numerical expression, recalling that Eq. (10) was derived using cgs units is crucial. Since water has a large dielectric constant and the dipole moment contribution is large in magnitude, this is actually a very tight upper bound. If instead we use the experimental value of $\epsilon_{w}=78$, we find $U_{1}^{q} / N=-118.4 \frac{\mathrm{kJ}}{\mathrm{mol}} \cdot \AA^{3} / \sigma^{3}$ for $T=300 \mathrm{~K}$ with variation lying within error bars of the simulation calculation of $U_{0}$. In Fig. 1 we used the infinite dielectric constant in our calculation of the energy correction.

As seen in Fig. 1, the inclusion of this correction brings all of the energies from Gaussian-truncated simulations much closer to the Ewald energy, shown as a horizontal line. All energies now lie well within $1 \%$ deviation from the Ewald energy, some with substantially less error than that, whereas only the three larger $\sigma$-values without $U_{1}^{q}$ would lie within the less stringent $5 \%$ deviation suggested as sufficient in Ref. 11 .

For solutions of charged particles, previous researchers obtained similar correction terms for energies, though their physical basis was less transparent ${ }^{5.30}$. The corrections by Hummer and coworkers relied on an analogy with the self-interaction in Ewald summations. The corrections by Wolf and coworkers drew upon the known limiting behaviors for charged fluids based on the StillingerLovett moment conditions. But the necessary extension to mixed charged and polar molecular systems was not appreciated. Combining thermodynamic perturbation theory with an examination of moment conditions for molecules, as in this paper, clarifies the general principles involved, and immediately leads to substantially improved energetics with a simple analytical energy correction.

\section{ANALYTICAL PRESSURE CORRECTION FOR NVT AND NPT SIMULATIONS}

Deriving a similar correction for the pressure may seem more problematic, since the pressure cannot be exactly expressed using only site-site distribution functions 31 , and to our knowledge no analytic pressure corrections have ever been suggested. However, LMF theory provides a general perspective that allows us to arrive at simple pressure corrections as well. To that end, we express the pressure thermodynamically as

$$
P=T\left(\frac{\partial S}{\partial V}\right)_{T,\left\{N_{M}\right\}}-\left(\frac{\partial U}{\partial V}\right)_{T,\left\{N_{M}\right\}} .
$$

Since our Gaussian-truncated system with purely 


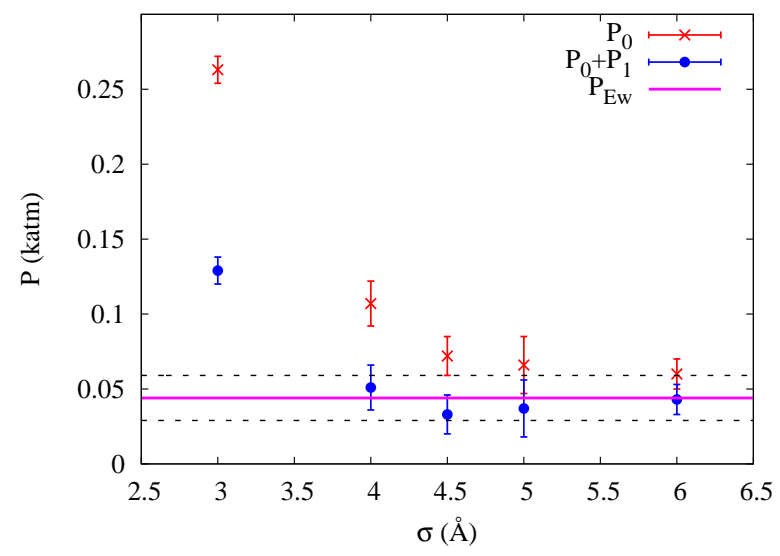

FIG. 3: Plot of pressure without ( $P_{0}$ shown in red crosses) and with the long-range correction $\left(P_{0}+P_{1}\right.$ shown in blue circles $)$ for the full range of truncation scales $\sigma$ studied. Error bars are shown for the data points, and error bars on the Ewald pressure are indicated by the thin dashed lines above and below the thick horizontal line at $0.044 \mathrm{katm}$.

short-ranged interactions reasonably captures the local order and structural variations expected to dominate the entropy, we expect that $S \approx S_{0}$ to a very good approximation, and we use this in the first term on the right in Eq. (12). Corrections from the long-ranged part of the Coulomb interactions to the pressure $P_{0}$ obtained directly from the truncated model simulation then arise from the second term and are simply related to a partial derivative of $U_{1}^{q}$ with respect to volume.

Using Eq. (10) to express $U_{1}^{q}$ in terms of $\left\{N_{M}\right\}, V$, and $T$, we find for rigid molecules that only the contribution due to dielectric shielding depends on volume. Therefore, regardless of the site composition of the rigid species, we have

$$
P_{1}^{q}=-\left(\frac{\partial U_{1}^{q}}{\partial V}\right)_{T,\left\{N_{M}\right\}}=-\frac{\mathrm{k}_{\mathrm{B}} \mathrm{T}}{2 \pi^{3 / 2} \sigma^{3}} \frac{\epsilon-1}{\epsilon} .
$$

This correction term is purely negative, just as we can deduce from our simulated $P_{0}$ for water shown in Fig. 3. Using the experimental dielectric constant of water, $\epsilon_{w}=78$, we find $P_{1}^{q}=-3.624 \mathrm{katm} \cdot \AA^{3} / \sigma^{3}$. As shown in Fig. 3, including $P_{1}^{q}$ brings nearly all pressures into agreement with the Ewald result. For flexible molecules, $\mu_{N}^{2},\left\langle l^{2}\right\rangle$, or $\alpha_{N}$ could have a volume dependence leading to a contribution to $P_{1}^{q}$. However, for dense and relatively incompressible systems, such a contribution is likely quite small.

This analytical pressure correction also proves useful for NPT simulations of the Gaussian-truncated water.

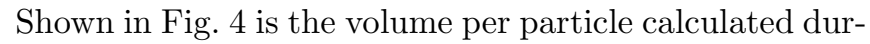
ing NPT simulations carried out at $300 \mathrm{~K}$ and $1 \mathrm{~atm}$. The spherically-truncated water simulations with $P=1 \mathrm{~atm}$ have a higher volume per particle than the Ewald results as found in Ref. 7. However when Gaussian-truncated water is simulated with a corrected external pressure adjusted to be $P_{\text {ext }}=P_{0}=1 \mathrm{~atm}-P_{1}^{q}(T, \sigma)$, the average

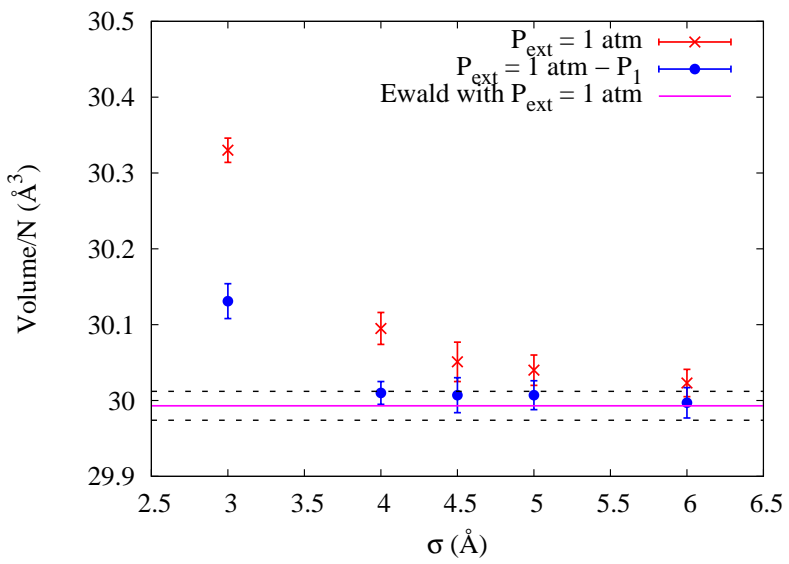

FIG. 4: Plots of the volume per particle determined from NPT simulation using $v_{0}(r)$. Error bars are as in Fig. 3 Applying the $P_{1}^{q}$ correction as an external pressure brings particle volumes in the mimic system into better agreement with the Ewald result.

volume per particle agrees quite well with Ewald results for all $\sigma$ but the smallest of $3.0 \AA$. The latter discrepency simply indicates that the second order $k$-space expansion for $\hat{\rho}^{q q}$ is insufficient for the smallest $\sigma$ used.

\section{CONCLUDING REMARKS}

In general, as has been well established $\frac{18,19}{}$, despite the highly accurate local structures obtained when using reasonable spherical truncations, the impact of the longranged forces on thermodynamics cannot be neglected. We have shown here that high accuracy is possible for energy, pressure, and density in spherically-truncated simulations of bulk molecular fluids solely by using simple, analytical corrections.

Thermodynamic corrections for nonuniform systems treated via LMF theory will be less straightforward. For example, for many of the slab systems we have simulated, a self-consistent $\mathcal{V}_{\mathrm{R}}$ is crucial for the structure ${ }^{15,16}$, and the full LMF theory should be used for the thermodynamics as well. Corrections to thermodynamics from a strong-coupling simulation could perhaps be found in some cases based on the Carnie-Chan sum rules 29 , a nonuniform analog of the Stillinger-Lovett moment conditions, but further theoretical development is necessary. However the simple analytical corrections presented herein should be immediately useful in correcting the thermodynamics of many bulk systems of interest.

This work was supported by NSF grants CHE0517818 and CHE0848574. JMR acknowledges the support of the University of Maryland Chemical Physics fellowship. 


\section{APPENDIX A: DERIVATION OF ZEROTH AND SECOND MOMENT CONDITIONS FOR A MIXTURE OF NEUTRAL AND CHARGED SITE-SITE MOLECULES}

In this Appendix we define the two-point intermolecular charge-density function $\rho^{q q}\left(\mathbf{r}, \mathbf{r}^{\prime}\right)$ used to determine the total Coulomb energy and connect its behavior at small wave vectors to that of the fundamental chargecharge linear response function used in the theory of the dielectric constant, as discussed in Appendix B. These results are used in the main body to derive corrections to the thermodynamics of uniform site-site molecular models simulated with spherically truncated Coulomb interactions.

The total Coulomb energy obtained during simulation of a small site-site molecular species without any intramolecular charge-charge interactions is

$$
\begin{aligned}
& U^{q}=\left\langle\frac{1}{2} \sum_{M}\right. \sum_{M^{\prime}} \sum_{i=1}^{N_{M}} \sum_{j=1}^{N_{M^{\prime}}}\left(1-\delta_{M M^{\prime}} \delta_{i j}\right) \\
&\left.\times \sum_{\alpha=1}^{n_{M}} \sum_{\gamma=1}^{n_{M^{\prime}}} \frac{q_{\alpha M} q_{\gamma M^{\prime}}}{\mathbf{r}_{i M}^{(\alpha)}-\mathbf{r}_{j M^{\prime}}^{(\gamma)}}\right\rangle .
\end{aligned}
$$

In this notation, the angular brackets indicate a normalized ensemble average, $M$ and $M^{\prime}$ indicate a given molecular species, $i$ and $j$ indicate a given molecule of a given species, and $\alpha$ and $\gamma$ represent the intramolecular sites ${ }^{23,28}$. The Kronecker deltas are necessary to exclude any charge-charge interactions between intramolecular sites within a given molecule. This energy $U^{q}$ can be more compactly represented as

$$
U^{q}=\frac{1}{2} \int d \mathbf{r} \int d \mathbf{r}^{\prime} \frac{\rho^{q q}\left(\mathbf{r}, \mathbf{r}^{\prime}\right)}{\left|\mathbf{r}-\mathbf{r}^{\prime}\right|}
$$

where $\rho^{q q}$ is a two-point intermolecular charge-density function that explicity excludes any purely intramolecular charge correlations, as implied by Eq. (A1) and detailed below.

Comparing Eqs. (A1) and (A2), we see the composite function $\rho^{q q}\left(\mathbf{r}, \mathbf{r}^{\prime}\right)$ is a charge-weighted linear combination of all intermolecular, two-point site-site distribution functions 23,28 :

$$
\rho^{q q}\left(\mathbf{r}, \mathbf{r}^{\prime}\right) \equiv \sum_{\alpha M} \sum_{\gamma M^{\prime}} q_{\alpha M} q_{\gamma M^{\prime}} \rho_{\alpha M \gamma M^{\prime}}\left(\mathbf{r}, \mathbf{r}^{\prime}\right)
$$

For our purposes here, it is more useful to relate this function to the basic charge-charge linear response function used in the theory of the dielectric constant.

For solutions of primitive model ions, Stillinger and Lovett showed that charge neutrality and screening place specific requirements on the behavior of the charge density in $k$-space at small wave vectors ${ }^{24}$. More generally, for a fluid composed of charged and polar molecules, the dielectric screening behavior of the molecules places restrictions on the decay of the two-point charge density $\rho^{q q}$. Based on this observation, we are able to harness a theoretical development of Chandler ${ }^{23}$ that expresses the dielectric constant $\epsilon$ of polar molecules in terms of an exact sum of charge-density-weighted site pair correlation functions. We generalize the derivation to include both charged and neutral site-site molecules and we take the dielectric constant as a given. From this vantage point, we may instead use these relations to place requirements on the decay of the two-point charge density $\rho^{q q}$.

We first define the instantaneous single-point total charge-density $\rho^{q}(\mathbf{r}, \overline{\mathbf{R}})$, a function of both a given external position $\mathbf{r}$ and the set $\overline{\mathbf{R}} \equiv\left\{\mathbf{R}_{i M}\right\} \equiv\left\{\mathbf{r}_{i M}^{(\alpha)}\right\}$ of positions of all mobile charged sites in a given configuration, as

$$
\rho^{q}(\mathbf{r}, \overline{\mathbf{R}}) \equiv \sum_{M} \sum_{i=1}^{N_{M}} \sum_{\alpha=1}^{n_{M}} q_{\alpha M} \delta\left(\mathbf{r}-\mathbf{r}_{i M}^{(\alpha)}\right) .
$$

With such a definition, the ensemble-averaged charge density profile $\rho^{q}(\mathbf{r})$ is

$$
\rho^{q}(\mathbf{r})=\left\langle\rho^{q}(\mathbf{r}, \overline{\mathbf{R}})\right\rangle .
$$

In the case of a uniform system, $\rho^{q}(\mathbf{r})=0$.

Comparing Eqs. (A1) and (A22) and using Eq. (A44), we may also express the two point charge function $\rho^{q q}\left(\left|\mathbf{r}-\mathbf{r}^{\prime}\right|\right)$ for a uniform system as

$$
\begin{aligned}
& \rho^{q q}\left(\left|\mathbf{r}-\mathbf{r}^{\prime}\right|\right)=\left\langle\rho^{q}(\mathbf{r}, \overline{\mathbf{R}}) \rho^{q}\left(\mathbf{r}^{\prime}, \overline{\mathbf{R}}\right)\right\rangle \\
& \quad-\left\langle\sum_{M} N_{M} \sum_{\alpha=1}^{n_{M}} \sum_{\gamma=1}^{n_{M}} q_{\alpha M} q_{\gamma M} \delta\left(\mathbf{r}-\mathbf{r}_{1 M}^{(\alpha)}\right) \delta\left(\mathbf{r}^{\prime}-\mathbf{r}_{1 M}^{(\gamma)}\right)\right\rangle .
\end{aligned}
$$

We have used the equivalence of all molecules of type $M$ in the last term. This term removes purely intramolecular charge-density correlations; we shall determine the small- $k$ contributions from this term based on well-known molecular properties using the approach of Chandler ${ }^{23}$ later in this appendix.

The first term, in contrast, is exactly the charge-charge linear response function for a uniform neutral system:

$$
\begin{aligned}
\left\langle\rho^{q}(\mathbf{r}, \overline{\mathbf{R}}) \rho^{q}\left(\mathbf{r}^{\prime}, \overline{\mathbf{R}}\right)\right\rangle & =\left\langle\delta \rho^{q}(\mathbf{r}, \overline{\mathbf{R}}) \delta \rho^{q}\left(\mathbf{r}^{\prime}, \overline{\mathbf{R}}\right)\right\rangle \\
& =\chi^{q q}\left(\left|\mathbf{r}-\mathbf{r}^{\prime}\right|\right) .
\end{aligned}
$$

Here $\delta \rho^{q}(\mathbf{r}, \overline{\mathbf{R}}) \equiv \rho^{q}(\mathbf{r}, \overline{\mathbf{R}})-\left\langle\rho^{q}(\mathbf{r}, \overline{\mathbf{R}})\right\rangle$. Physically $\chi^{q q}$ describes the coupling between charge-density fluctuations at positions $\mathbf{r}$ and $\mathbf{r}^{\prime}$. As is well established 20,23 , such a function is intimately related to the dielectric behavior of the fluid at long distances, and furthermore, may be easily analyzed based on basic electrostatics and standard definitions of the functional derivative. This relationship is discussed in Appendix B.

Our goal is to write a small- $k$ expansion of the twopoint intermolecular charge density,

$$
\hat{\rho}^{q q}(k) \approx \hat{\rho}^{(0) q q}+k^{2} \hat{\rho}^{(2) q q}+\mathcal{O}\left(k^{4}\right),
$$


where from Eqs. (A6) and (A7)

$$
\begin{aligned}
\rho^{q q}(\mid \mathbf{r} & \left.-\mathbf{r}^{\prime} \mid\right)=\chi^{q q}\left(\left|\mathbf{r}-\mathbf{r}^{\prime}\right|\right) \\
& -\left\langle\sum_{M} N_{M} \sum_{\alpha=1}^{n_{M}} \sum_{\gamma=1}^{n_{M}} q_{\alpha M} q_{\gamma M} \delta\left(\mathbf{r}-\mathbf{r}_{1 M}^{(\alpha)}\right) \delta\left(\mathbf{r}^{\prime}-\mathbf{r}_{1 M}^{(\gamma)}\right)\right\rangle .
\end{aligned}
$$

As shown in Appendix B, the charge-charge linear response function $\hat{\chi}^{q q}$ may be expanded as

$$
\hat{\chi}^{q q}(k)=0+\frac{\mathrm{k}_{\mathrm{B}} \mathrm{T}}{4 \pi}\left(1-\frac{1}{\epsilon}\right) k^{2}+\mathcal{O}\left(k^{4}\right) .
$$

Now we must remove the intramolecular contributions as described by the last term in Eq. (A9). Defining the conditional singlet intramolecular site density functions $\varrho_{\alpha \mid \gamma M}\left(\mathbf{r} \mid \mathbf{r}^{\prime}\right)$ for $\alpha \neq \gamma$ as

$$
\rho_{\gamma M}\left(\mathbf{r}^{\prime}\right) \varrho_{\alpha \mid \gamma M}\left(\mathbf{r} \mid \mathbf{r}^{\prime}\right)=\left\langle N_{M} \delta\left(\mathbf{r}-\mathbf{r}_{1 M}^{(\alpha)}\right) \delta\left(\mathbf{r}^{\prime}-\mathbf{r}_{1 M}^{(\gamma)}\right)\right\rangle,
$$

and applying consequences of uniformity, Eq. (A99) can be written as

$$
\begin{aligned}
& \rho^{q q}\left(\left|\mathbf{r}-\mathbf{r}^{\prime}\right|\right)=\chi^{q q}\left(\left|\mathbf{r}-\mathbf{r}^{\prime}\right|\right) \\
& \quad-\sum_{M} \rho_{M} \sum_{\alpha, \gamma} q_{\alpha M} q_{\gamma M} \omega_{\alpha \gamma M}\left(\left|\mathbf{r}-\mathbf{r}^{\prime}\right|\right),
\end{aligned}
$$

where

$$
\omega_{\alpha \gamma M}\left(\left|\mathbf{r}-\mathbf{r}^{\prime}\right|\right) \equiv \delta_{\alpha \gamma} \delta\left(\mathbf{r}-\mathbf{r}^{\prime}\right)+\varrho_{\alpha \mid \gamma M}\left(\left|\mathbf{r}-\mathbf{r}^{\prime}\right|\right) .
$$

For neutral molecules, Chandler demonstrated that the small- $k$ components of $\hat{\omega}_{\alpha \gamma M}(k)$ are related to simple properties of the molecule. For both charged and uncharged molecules, the zeroth moment of $\hat{\omega}_{\alpha \gamma M}$ is simply

$$
\begin{aligned}
\hat{\omega}_{\alpha \gamma M}^{(0)} & =\delta_{\alpha \gamma}+\int d \mathbf{r} \varrho_{\alpha \mid \gamma M}(\mathbf{r}) \\
& =\delta_{\alpha \gamma}+\left(1-\delta_{\alpha \gamma}\right)=1 .
\end{aligned}
$$

Using this exact expression in Eq. A12 yields

$$
\hat{\rho}^{(0) q q}=\hat{\chi}^{(0) q q}-\sum_{M} \rho_{M} \sum_{\alpha, \gamma} q_{\alpha M} q_{\gamma M}=-\sum_{M} \rho_{M} q_{M}^{2},
$$

an expression encompassing the standard zeroth moment condition for ions ${ }^{24}$ and the zeroth moment for neutral molecular species $\underline{23}$.

The expression for $\hat{\omega}_{M}^{(2)}$ determined by Chandler ${ }^{23}$ may be written most generally as

$$
\begin{aligned}
\hat{\omega}_{M}^{(2)} & \equiv \sum_{\alpha \neq \gamma} q_{\alpha M} q_{\gamma M} \hat{\omega}_{\alpha \gamma M}^{(2)} \\
& =-\frac{1}{6} \int d \mathbf{r} \sum_{\alpha \neq \gamma} q_{\alpha M} q_{\gamma M} \varrho_{\alpha \mid \gamma M}(\mathbf{r}) r^{2} \\
& =-\frac{1}{6} \sum_{\alpha \neq \gamma} q_{\alpha M} q_{\gamma M}\left\langle l_{\alpha \gamma M}^{2}\right\rangle,
\end{aligned}
$$

where $l_{\alpha \gamma M}$ is the bondlength between sites $\alpha$ and $\gamma$ for a molecule of species $M$. As shown in Ref. 23, for a neutral molecule indicated by $N$ below, the final summation in the above equation is simply related to the molecular dipole moment $\mu_{N}$ and the molecular polarizability $\alpha_{N}$ as

$$
\hat{\omega}_{N}^{(2)}=\sum_{\alpha \neq \gamma} q_{\alpha N} q_{\gamma N} \hat{\omega}_{\alpha \gamma N}^{(2)}=\frac{1}{3} \mu_{N}^{2}+\mathrm{k}_{\mathrm{B}} \mathrm{T} \alpha_{N} .
$$

This relationship does not hold for a charged molecule since the dipole moment then depends on the choice of coordinate system.

Distinguishing charged species $(C)$ and neutral species $(N)$ where $\{M\}=\{N\} \cup\{C\}$, and without substituting for $\hat{\omega}_{N}^{(2)}$ and $\hat{\omega}_{C}^{(2)}$, we find

$$
\begin{aligned}
\hat{\rho}^{(2) q q} & =\frac{\mathrm{k}_{\mathrm{B}} \mathrm{T}}{4 \pi} \frac{\epsilon-1}{\epsilon}-\sum_{M} \rho_{M} \sum_{\alpha \neq \gamma} q_{\alpha M} q_{\gamma M} \hat{\omega}_{\alpha \gamma M}^{(2)} \\
& =\frac{\mathrm{k}_{\mathrm{B}} \mathrm{T}}{4 \pi} \frac{\epsilon-1}{\epsilon}-\sum_{N} \rho_{N} \hat{\omega}_{N}^{(2)}-\sum_{C} \rho_{C} \hat{\omega}_{C}^{(2)} .
\end{aligned}
$$

Thus, we may write a general expression for $\hat{\rho}^{q q}$ in $k$ space. Utilizing the expressions for $\hat{\omega}_{N}^{(2)}$ and $\hat{\omega}_{C}^{(2)}$, we have

$$
\begin{aligned}
\hat{\rho}^{q q}(k)= & -\sum_{C} \rho_{C} q_{C}^{2}+k^{2} \frac{\mathrm{k}_{\mathrm{B}} \mathrm{T}}{4 \pi} \frac{\epsilon-1}{\epsilon} \\
& -k^{2} \sum_{N} \rho_{N}\left\{\frac{1}{3} \mu_{N}^{2}+\mathrm{k}_{\mathrm{B}} \mathrm{T} \alpha_{N}\right\} \\
& +k^{2} \frac{1}{6} \sum_{C} \rho_{C} \sum_{\alpha \neq \gamma} q_{\alpha C} q_{\gamma C}\left\langle l_{\alpha \gamma C}^{2}\right\rangle+\mathcal{O}\left(k^{4}\right) .
\end{aligned}
$$

Unlike the simple expansion of $\hat{\chi}^{q q}(k)$ in Eq. (A10), we see that the small- $k$ behavior of $\hat{\rho}^{q q}(k)$ depends on several simple properties of the solution as a whole, like the dipole moment and polarizability of individual neutral molecules, and the net molecular charge and the average square bond lengths of charged molecules, as well as the dielectric constant. Thus by knowing simple single molecule properties and the long wavelength dielectric constant, we know how intermolecular charge-charge correlations decay in solution. This is the essential idea used to develop energy and pressure corrections for simulations of bulk liquids using molecular models with truncated Coulomb interactions.

A related expression may be developed for larger molecular species with intramolecular charge-charge interactions given by CHARMM- Or AMBER-like molecular models by modifying the total Coulomb energy to solely exclude the charge-charge interactions of sites $i$ and $j$ within two bonded connections of one another, using a "bonding function" $B_{M}(i, j)$ that acts similar to the product of Kronecker deltas in Eq. (A1). In such cases, the expansion of $\hat{\chi}^{q q}$ remains the same but the remaining 
contribution to $\hat{\rho}^{q q}$ may no longer be simply represented using only whole-molecule properties such as the dipole moment and polarizability.

\section{APPENDIX B: EXACT MOMENT CONDITIONS ON CHARGE-CHARGE LINEAR RESPONSE}

We present the following moment conditions for $\chi^{q q}$ distinct from the molecular-specific analysis found in Appendix A because the results are more general than the specific site-site molecules chosen. The analysis of the behavior of $\hat{\chi}^{q q}$ at small $k$ is similar to that found in References 20 and 23 and the final results are identical. Our derivation is simpler because we focus directly on the total charge density; this also allows us to derive both the Stillinger-Lovett moment conditions for charged systems and the formula for the dielectric constant of a polar mixture from the same footing.

The electrostatic potential at $\mathbf{r}$ induced by a fixed external charge distribution $\rho_{\text {ext }}^{q}\left(\mathbf{r}^{\prime}\right)$ (e.g., a test charge $Q$ placed at the origin, as considered by Chandler $\left.{ }^{23}\right)$ is given by

$$
\mathcal{V}_{\mathrm{ext}}(\mathbf{r})=\int \frac{\rho_{\mathrm{ext}}^{q}\left(\mathbf{r}^{\prime}\right)}{\left|\mathbf{r}-\mathbf{r}^{\prime}\right|} d \mathbf{r}^{\prime}
$$

and the associated electrostatic energy for a particular microscopic configuration characterized by the set of molecular positions $\overline{\mathbf{R}}$ is then

$$
U_{\text {ext }}^{q}(\overline{\mathbf{R}})=\int \rho^{q}(\mathbf{r}, \overline{\mathbf{R}}) \mathcal{V}_{\text {ext }}(\mathbf{r}) d \mathbf{r} .
$$

Here $\rho^{q}(\mathbf{r}, \overline{\mathbf{R}})$ is the total configurational charge density, defined in the particular case of a mixture of site-site molecules by Eq. (A4). This energy contribution will appear in the nonuniform system's Hamiltonian when $\mathcal{V}_{\text {ext }}(\mathbf{r})$ is nonzero.

As such, we know from standard definitions of functional differentiation of free energies ${ }^{20,32}$ that

$$
\frac{\delta[-\beta A]}{\delta\left[-\beta \mathcal{V}_{\text {ext }}(\mathbf{r})\right]}=\left\langle\rho^{q}(\mathbf{r}, \overline{\mathbf{R}})\right\rangle_{\mathcal{V}} \equiv \rho_{\mathcal{V}}^{q}(\mathbf{r}),
$$

where $\beta \equiv\left(\mathrm{k}_{\mathrm{B}} \mathrm{T}\right)^{-1}$ and the subscript $\mathcal{V}$ indicates that the ensemble average is taken in the presence of an external potential. Similarly we have

$$
\begin{aligned}
\frac{\delta \rho_{\mathcal{V}}^{q}(\mathbf{r})}{\delta\left[-\beta \mathcal{V}_{\mathrm{ext}}\left(\mathbf{r}^{\prime}\right)\right]} & =\frac{\delta[-\beta A]}{\delta\left[-\beta \mathcal{V}_{\mathrm{ext}}(\mathbf{r})\right] \delta\left[-\beta \mathcal{V}_{\mathrm{ext}}\left(\mathbf{r}^{\prime}\right)\right]} \\
& =\chi_{\mathcal{V}}^{q q}\left(\mathbf{r}, \mathbf{r}^{\prime}\right)
\end{aligned}
$$

The total electrostatic potential at position $\mathbf{r}$ in the nonuniform fluid is then given by the sum of the external potential and the induced polarization potential:

$$
\begin{aligned}
\mathcal{V}_{\text {tot }}(\mathbf{r}) & =\mathcal{V}_{\text {ext }}(\mathbf{r})+\mathcal{V}_{\text {pol }}(\mathbf{r}) \\
& =\mathcal{V}_{\text {ext }}(\mathbf{r})+\int d \mathbf{r}^{\prime} \frac{\rho_{\mathcal{V}}^{q}\left(\mathbf{r}^{\prime}\right)}{\left|\mathbf{r}-\mathbf{r}^{\prime}\right|} .
\end{aligned}
$$

To get a formula for the dielectric constant we expand about the uniform neutral system and evaluate $\mathcal{V}_{\text {pol }}$ to linear order in $\mathcal{V}_{\text {ext }}$ using Eq. (B4). This gives

$$
\begin{aligned}
\mathcal{V}_{\mathrm{pol}}(\mathbf{r}) & \approx \int \frac{d \mathbf{r}^{\prime}}{\left|\mathbf{r}-\mathbf{r}^{\prime}\right|} \int d \mathbf{r}^{\prime \prime} \frac{\delta \rho_{\mathcal{V}}^{q}\left(\mathbf{r}^{\prime}\right)}{\delta\left[-\beta \mathcal{V}_{\mathrm{ext}}\left(\mathbf{r}^{\prime \prime}\right)\right]}\left[-\beta \mathcal{V}_{\mathrm{ext}}\left(\mathbf{r}^{\prime \prime}\right)\right] \\
& =-\int \frac{d \mathbf{r}^{\prime}}{\left|\mathbf{r}-\mathbf{r}^{\prime}\right|} \int d \mathbf{r}^{\prime \prime} \beta \chi^{q q}\left(\left|\mathbf{r}^{\prime}-\mathbf{r}^{\prime \prime}\right|\right) \mathcal{V}_{\mathrm{ext}}\left(\mathbf{r}^{\prime \prime}\right) .
\end{aligned}
$$

Here $\chi^{q q}$ is the linear response function in the uniform fluid as in Eq. (A7). Taking the Fourier transform of the final equation, we find

$$
\hat{\mathcal{V}}_{\text {tot }}(\mathbf{k})=\hat{\mathcal{V}}_{\text {ext }}(\mathbf{k})-\frac{4 \pi}{k^{2}} \beta \hat{\chi}^{q q}(k) \hat{\mathcal{V}}_{\text {ext }}(\mathbf{k})
$$

Thus to linear order we have

$$
\frac{\hat{\mathcal{V}}_{\text {tot }}(\mathbf{k})}{\hat{\mathcal{V}}_{\text {ext }}(\mathbf{k})}=1-\frac{4 \pi \beta}{k^{2}} \hat{\chi}^{q q}(k)
$$

Phenomenologically, we know that in the limit of $\mathbf{k} \rightarrow$ 0 , this ratio of the total electrostatic potential to the externally-imposed potential is exactly $1 / \epsilon$. Therefore, we find for our molecular mixture the general result

$$
\lim _{\mathbf{k} \rightarrow 0}\left(1-\frac{4 \pi \beta}{k^{2}} \hat{\chi}^{q q}(k)\right)=\frac{1}{\epsilon} .
$$

Based on the limit in Eq. (B9), and expanding $\hat{\chi}^{q q}$ for small $k$ as $\hat{\chi}^{(0) q q}+\hat{\chi}^{(2) q q} k^{2}$, we have

$$
\begin{aligned}
\hat{\chi}^{(0) q q} & =0 \\
4 \pi \beta \hat{\chi}^{(2) q q} & =1-\frac{1}{\epsilon} .
\end{aligned}
$$

Any mixture with mobile ions acts as a conductor with $\epsilon=\infty$ in Eq. (B10), independent of the nature of the neutral components, and these results then reduce to the Stillinger-Lovett moment conditions ${ }^{24}$.

\footnotetext{
* Present address: Materials Science Division, Lawrence Berkeley National Laboratory, Berkeley, CA 94720

† Electronic address: jdw@ipst.umd.edu
}

1 Y. Duan, C. Wu, S. Chowdhury, M. Lee, G. Xiong, W. Zhang, R. Yang, P. Cieplak, R. Luo, T. Lee, et al., J. Comp. Chem. 24, 1999 (2003). 
2 J. A. D. MacKerell, D. Bashford, M. Bellott, J. R. L. Dunbrack, J. D. Evanseck, M. J. Field, S. Fischer, J. Gao, H. Guo, S. Ha, et al., J. Phys. Chem. B 102, 3586 (1998).

3 D. Frenkel and B. Smit, Understanding Molecular Simulation: From Algorithms to Applications (Academic Press, New York, 2002), 2nd ed.

${ }^{4}$ C. Fennell and J. Gezelter, J. Chem. Phys. 124, 234104 (2006).

5 D. Wolf, P. Keblinski, S. Phillpot, and J. Eggebrecht, J. Chem. Phys. 110, 8254 (1999).

6 I. Nezbeda, Mol. Phys. 103, 59 (2005).

7 S. Izvekov, J. M. J. Swanson, and G. A. Voth, J. Phys. Chem. A 112, 4711 (2008).

8 G. Hummer, D. Soumpasis, and M. Neumann, J. Phys. Condens. Matt. 6, A141 (1994).

9 S. E. Feller, R. W. Pastor, A. Rojnuckarin, S. Bogusz, and B. R. Brooks, J. Phys. Chem. 100, 17011 (1996).

${ }^{10}$ E. Spohr, J. Chem. Phys. 107, 6342 (1997).

11 D. Zahn, B. Schilling, and S. Kast, J. Phys. Chem. B 106, 10725 (2002).

12 J. D. Weeks, K. Katsov, and K. Vollmayr, Phys. Rev. Lett. 81, 4400 (1998).

13 J. M. Rodgers and J. D. Weeks, J. Phys. - Condens. Matt. 20, 494206 (2008).

14 Y. G. Chen and J. D. Weeks, Proc. Nat. Acad. Sci. USA 103, 7560 (2006).

15 J. M. Rodgers, C. Kaur, Y.-G. Chen, and J. D. Weeks, Phys. Rev. Lett. 97, 097801 (2006).

16 J. M. Rodgers and J. D. Weeks, Proc. Nat. Acad. Sci. USA 105, 19136 (2008).
17 J. M. Rodgers and J. D. Weeks (in preparation).

18 Y. G. Chen, C. Kaur, and J. D. Weeks, J. Phys. Chem. B 108, 19874 (2004).

19 N. A. Denesyuk and J. D. Weeks, J. Chem. Phys. 128, 124109 (2008).

20 J.-P. Hansen and I. R. McDonald, Theory of Simple Liquids (Academic Press, New York, 2006), 3rd ed.

21 H. Berendsen, J. Grigera, and T. Straatsma, J. Phys. Chem. 91, 6269 (1987).

22 Z. Hu, J. M. Rodgers, and J. D. Weeks (in preparation).

23 D. Chandler, J. Chem. Phys. 67, 1113 (1977).

${ }^{24}$ F. H. Stillinger and R. Lovett, J. Chem. Phys. 49, 1991 (1968).

25 D. M. Ceperley and C. V. Chester, Phys. Rev. A 15, 755 (1977); P. Linse and H. C. Andersen, J. Chem. Phys. 85, 3027 (1986).

26 W. Smith, Mol. Sim. 32, 933 (2006).

27 H. J. C. Berendsen, J. P. M. Postma, W. F. van Gunsteren, A. Dinola, and J. R. Haak, J. Chem. Phys. 81, 3684 (1984).

28 D. Chandler and L. R. Pratt, J. Chem. Phys. 65, 2925 (1976).

29 P. Martin, Rev. Mod. Phys. 60, 1075 (1988).

30 G. Hummer, L. Pratt, and A. Garcia, J. Phys. Chem. 100, 1206 (1996).

31 R. Topol and P. Claverie, Mol. Phys. 35, 1753 (1978); D. Chandler in The Liquid State of Matter: Fluids, Simple and Complex (North Holland, New York, 1982), p. 275.

32 J. K. Percus, in The Equilibrium Theory of Classical Fluids (W. A. Benjamin, Inc., New York, 1964), p. II-33. 